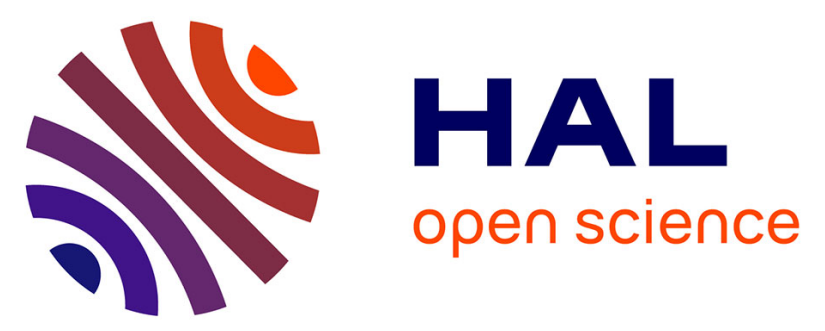

\title{
Analyse séquentielle 3D de la cinématique fémoropatellaire sur genou normal à partir de radiographies biplanaires : protocole de validation in vitro
}

Louis Dagneaux, Patricia Thoreux, Boris Eustache, François Canovas, Wafa

Skalli

\section{To cite this version:}

Louis Dagneaux, Patricia Thoreux, Boris Eustache, François Canovas, Wafa Skalli. Analyse séquentielle 3D de la cinématique fémoropatellaire sur genou normal à partir de radiographies biplanaires : protocole de validation in vitro. Revue de Chirurgie Orthopédique et Traumatologique, 2015, 101 (7), pp.534-541. 10.1016/j.rcot.2015.09.304 . hal-03267653

\section{HAL Id: hal-03267653 https://hal.science/hal-03267653}

Submitted on 22 Jun 2021

HAL is a multi-disciplinary open access archive for the deposit and dissemination of scientific research documents, whether they are published or not. The documents may come from teaching and research institutions in France or abroad, or from public or private research centers.
L'archive ouverte pluridisciplinaire HAL, est destinée au dépôt et à la diffusion de documents scientifiques de niveau recherche, publiés ou non, émanant des établissements d'enseignement et de recherche français ou étrangers, des laboratoires publics ou privés. 


\title{
Analyse séquentielle 3D de la cinématique fémoropatellaire sur genou normal à partir de radiographies biplanaires : protocole de validation in vitro
}

\author{
Sequential 3D analysis of patellofemoral kinematics from biplanar X-rays: In vitro \\ validation protocol
}

\author{
L. Dagneaux ${ }^{\mathrm{a}, *, \mathrm{~b}}$, P. Thoreux ${ }^{\mathrm{a}, \mathrm{c}}$, B. Eustache ${ }^{\mathrm{a}}$, F. Canovas ${ }^{\mathrm{b}}$, W. Skalli ${ }^{\mathrm{a}}$ \\ a Institut de biomécanique humaine Georges-Charpak, arts et métiers ParisTech (ENSAM), 151, boulevard de l'Hôpital, 75013 Paris, France \\ ${ }^{\mathrm{b}}$ Département de chirurgie orthopédique et traumatologie, unité de chirurgie du membre inférieur, hôpital Lapeyronie, CHRU de Montpellier, 371, avenue \\ Gaston-Giraud, 34295 Montpellier cedex 5, France \\ ${ }^{c}$ Hôpital Avicenne, université Paris 13, Sorbonne Paris-Cité, AP-HP, 93017 Bobigny, France
}

Mots clés :

Fémoropatellaire

Cinématique

Radiographies biplanaires

EOS

3D

In vitro

Instabilité

\section{R É S U M É}

Introduction. - Caractériser la fonction fémoropatellaire à l'aide de critères cinématiques est essentiel à sa compréhension, à son évaluation et à son suivi. Le but était d'évaluer une méthode d'analyse 3D séquentielle par radiographies biplanaires à partir d'un protocole in vitro.

Hypothèse. - L'utilisation d'un système d'imagerie biplanaire associé à de nouvelles méthodes de recons-truction 3D permet une analyse fémoropatellaire fiable, sans imagerie préalable.

Matériel et méthodes. - Huit pièces anatomiques ont été étudiées au cours de cycles de flexion de genou de 0 à $60^{\circ}$ par un simulateur in vitro. La validation du protocole comprenait une évaluation du mouvement séquentiel et continu à l'aide d'un système optoélectronique, une analyse de la précision et de la repro-ductibilité des mesures à partir de billes métalliques insérées dans la patella, ainsi qu'une vérification de la géométrie patellaire 3D comparativement à des acquisitions tomodensitométriques.

Résultats. - Les différences de position entre cinématique séquentielle et continue étaient inférieures à $1 \mathrm{~mm}$ et $1^{\circ}$. Certaines composantes de mouvement pouvaient être suivies de manière fiable : les transla-tions, la flexion et la bascule patellaires. Cette analyse rapportait une incertitude inférieure à $2 \mathrm{~mm}$ pour les translations et à $3^{\circ}$ pour les rotations, à l'exception de la rotation frontale. L'incertitude autour de la bascule patellaire était de $5^{\circ}$. La différence moyenne de géométrie était de $0,49 \mathrm{~mm}$.

Discussion. - L'analyse séquentielle est cohérente avec la cinématique continue. Cette méthode d'analyse fournit au clinicien des paramètres positionnels de la patella sans imagerie scanner ou IRM préalable. Une étude clinique reste à envisager pour l'identification de profils cinématiques fémoropatellaires et de critères positionnels in vivo.

Niveau de preuve. - IV (étude expérimentale).

\section{Introduction}

L'évaluation de la cinématique fémoropatellaire (FP) est difficile. La caractérisation qualitative et quantitative de la course

DOI de l'article original : http://dx.doi.org/10.1016/j.otsr.2015.07.017.

is Ne pas utiliser, pour citation, la référence française de cet article, mais celle de l'article original paru dans Orthopaedics \& Traumatology: Surgery \& Research, en utilisant le DOI ci-dessus.

* Auteur correspondant.

Adresse e-mail : louisdagneaux@gmail.com (L. Dagneaux). patellaire n'est pas réalisable en routine clinique, et manque à l'évaluation fonctionnelle des patients présentant des instabilités ou des syndromes douloureux patellaires. La relation entre anomalies cinématiques et troubles cliniques FP est d'ailleurs controversée faute de moyens d'investigation fiables et disponibles. Dans le cadre du syndrome douloureux patellaire, les différences cinématiques ou positionnelles sont variables en fonction des conditions de charge du membre inférieur [1], voire non retrouvées entre genoux symptomatiques et asymptomatiques [2,3]. Une récente revue de littérature suggère que ces anomalies ne sont que des facteurs de risques associés et qu'il convient de redéfinir avec 
précision la course patellaire normale [4]. L'identification des variations positionnelles de la patella nous paraît également essentielle à la compréhension de la fonction $\mathrm{FP}$, à son évaluation et à son suivi.

Afin de proposer de nouvelles techniques d'investigation in vivo, certaines équipes s'appuient sur des études de validation in vitro. Si les capteurs cutanés induisent des artefacts de déplacement entre la peau et les os [5], la plupart des protocoles publiés utilisent un système optoélectronique [6-8], magnétique [9-11] ou fluoroscopique [12,13]. Cependant, la plupart de ces méthodes nécessitent une imagerie tomodensitométrique préalable, afin d'utiliser une géométrie osseuse fiable lors des analyses cinématiques. Le récent développement de l'imagerie par radiographies biplanaires permet une analyse peu irradiante du squelette du membre inférieur en charge. Son utilisation a précédemment été évaluée dans l'analyse cinématique du genou [14,15], en y associant de nouvelles méthodes de référentiels et de reconstruction 3D [16,17].

Le but de ce travail était d'évaluer la précision et la reproductibilité d'un protocole séquentiel biplanaire fémoropatellaire à l'aide d'un simulateur automatisé de flexion de genou in vitro. L'hypothèse était que la cinématique FP peut être évaluée de manière fiable par un système d'imagerie biplanaire à l'aide de nouvelles méthodes de reconstruction.

\section{Matériel et méthodes}

\subsection{Pièces anatomiques}

Huit membres inférieurs ont été étudiés, provenant de quatre sujets cadavériques fraichement congelés (moins de 72 heures), âgés de 65 à 78 ans au moment du décès ( 2 femmes et 2 hommes, âge moyen 74 ans). Les prélèvements étaient réalisés après approbation du comité d'éthique du laboratoire d'anatomie des Saints-Pères (Institut d'anatomie, UFR biomédicale des SaintsPères, université René-Descartes, Paris). L'opérateur prélevait le membre inférieur par désarticulation coxo-fémorale et talo-crurale [14]. La conservation était réalisée par congélation à $-20^{\circ} \mathrm{C}$. Avant expérimentation, les pièces étaient décongelées à température ambiante pendant 12 heures. Deux groupes étaient constitués :

- quatre pièces anatomiques équipées de tripodes infrarouges [14], destinées à l'étude de la cinématique séquentielle ;

- quatre autres pièces avec billes métalliques enchâssées dans la patella (facette médiale, facette latérale et apex), destinées à l'étude de précision et reproductibilité.

\subsection{Simulateur de flexion du genou in vitro}

Le type de simulateur de flexion du genou à fémur fixe et tibia libre (Fig. 1) avait été validé au laboratoire lors d'une étude préliminaire de faisabilité [14]. Deux poids de 10 newtons étaient appliqués à l'extrémitédistale du tibia à l'aide d'un système de corde et poulies. La projection du point d'application correspondait au centre de la tête fémorale et la direction correspondait à l'axe fémoral mécanique. Les cycles de flexion-extension étaient générés à l'aide d'un vérin électrique (DSZY1, Drive-System Europ Ltd) dont l'action était appliquée au tendon quadricipital au moyen d'une corde en acier fixée par un clamp métallique (vitesse de traction de $12 \mathrm{~mm} / \mathrm{s}$ pour l'analyse continue).

\subsection{Protocole d'acquisition biplanaire et méthode d'interprétation $3 D$}

La pièce anatomique fixée sur le simulateur était installée dans la cabine du système d'imagerie (EOS, Eos Imaging, France). Ce

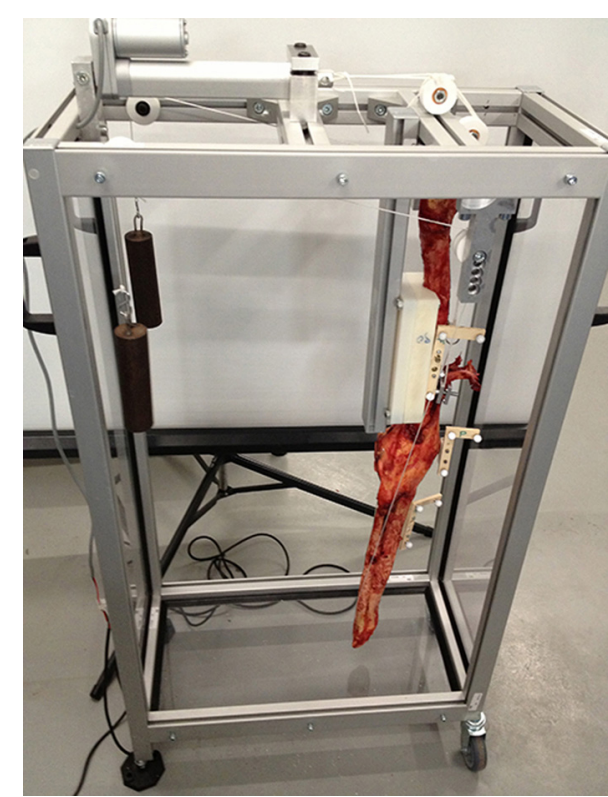

Fig. 1. Simulateur in vitro de flexion automatisée du genou.

protocole séquentiel consistait en une acquisition statique, biplanaire, calibrée et simultanée de chaque pièce anatomique dans 5 positions successives à $0^{\circ}, 20^{\circ}, 30^{\circ}, 45^{\circ}$ et $60^{\circ}$ de flexion du genou. La position en extension à $0^{\circ}$ (considérée comme position de référence) permettait l'acquisition manuelle par contourage de la géométrie osseuse du fémur, de la patella, des tripodes infrarouges ou des billes métalliques, afin de générer un modèle personnalisé par algorithme de reconstruction 3D (Fig. 2). Durant la phase dite " de recalage », l'opérateur faisait correspondre manuellement chaque objet $3 \mathrm{D}$ à ses contours respectifs sur les vues biplanaires suivantes. Le fémur étant fixe, aucune phase de recalage fémoral n'a été nécessaire. Fondé sur l'identification de régions spécifiques anatomiques, un système de coordonnées anatomiques pour chaque objet 3D (Fig. 3) permettait de quantifier leur position relative successive [14,17-19]. Un programme personnalisé (MATLAB V5R20, Mathworks, Natick, MA) permettait de calculer la position de la patella, des tripodes et des billes par rapport au référentiel fémur à chaque position de flexion du genou, à partir des composantes linéaires et angulaires issues des systèmes de coordonnées. La séquence d'angles $\left(\mathrm{Y}, \mathrm{X}^{\prime}, \mathrm{Z}^{\prime \prime}\right)$ était choisie pour le calcul de la matrice angulaire. La cinématique 3D de la patella était exprimée en 6 degrés de liberté, composés de 3 translations et 3 rotations (Fig. 4).

\section{4. Étapes de validation in vitro}

\subsubsection{Analyse du mouvement continu et séquentiel}

Dans le premier groupe de pièces anatomiques, les coordonnées 3D initiales de chaque tripode étaient préalablement enregistrées à l'aide d'un système de mesure optoélectronique (POLARIS ${ }^{\circledR}$ optoelectronic motion capture system, NDI, Waterloo, Ontario, Canada) par 2 caméras en position d'extension du genou, puis de manière continue jusqu'à $60^{\circ}$ de flexion (Fig. 5). Pour analyser l'homogénéité des cycles du simulateur, l'acquisition continue était réalisée pendant 6 cycles de flexion-extension successifs. Puis, on réalisait le protocole biplanaire dans un second temps avec les pièces munies de tripodes. Ainsi, l'analyse comprenait la comparaison au cours de la flexion des positions séquentielles des tripodes après recalage manuel et des positions continues optoélectroniques. 

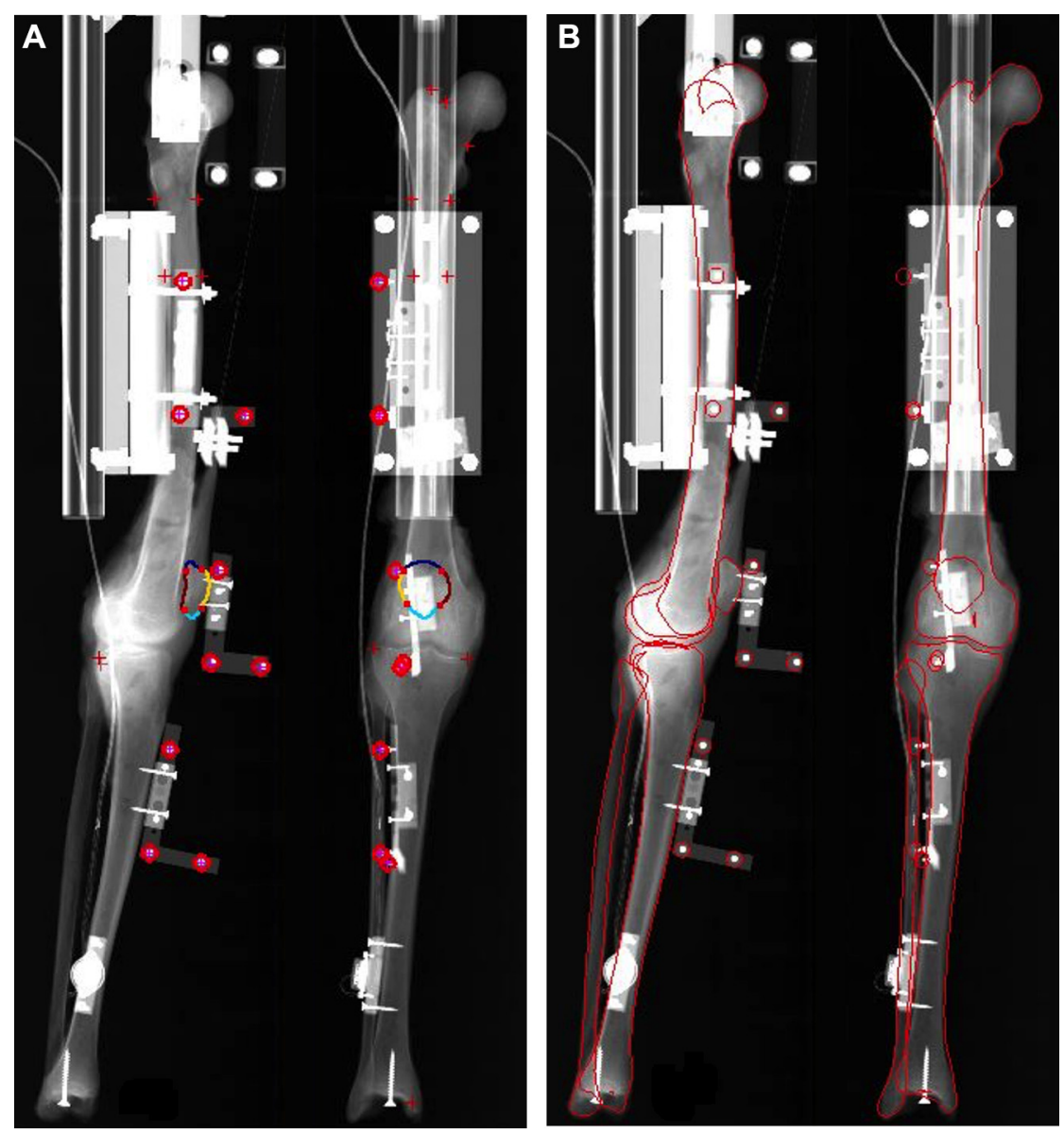

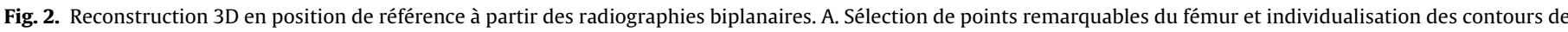
la patella et des tripodes. B. Reconstruction par algorithme 3D.
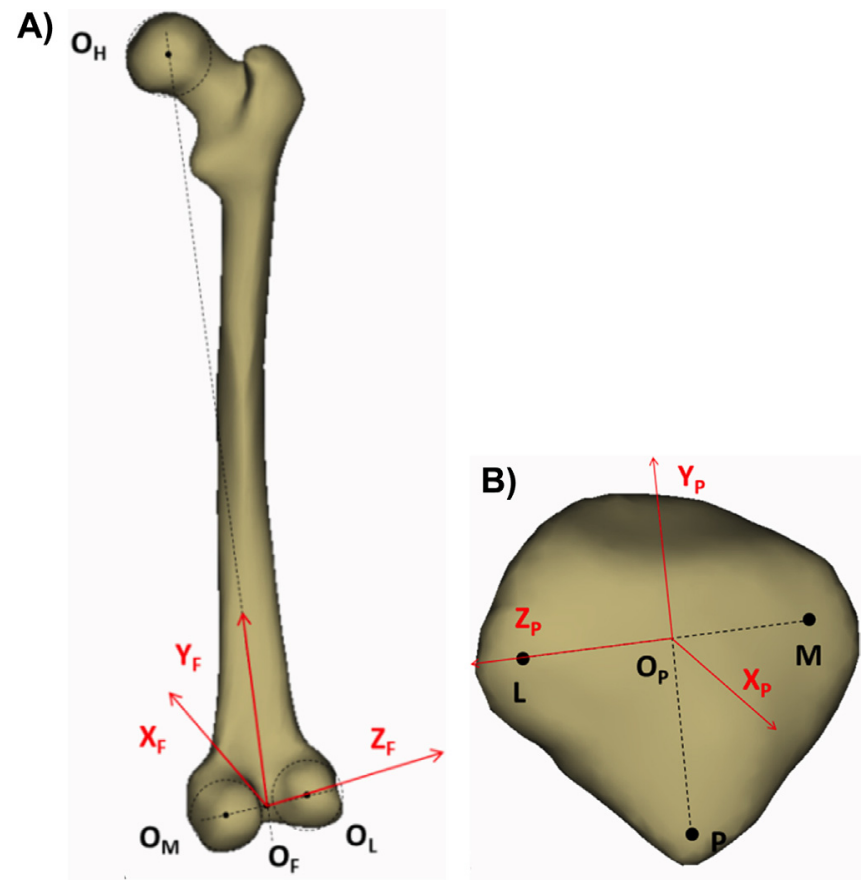

Fig. 3. Construction des repères anatomiques du fémur (A) et de la patella (B).

\subsubsection{Analyse de la géométrie osseuse par reconstruction biplanaire}

Les 4 pièces anatomiques du second groupe avaient eu au préalable une acquisition tomodensitométrique (Philips ${ }^{\circledR}$ ICT 256, service de radiologie polyvalente, hôpital La-Pitié-Salpêtrière, Paris, France) afin d'évaluer la géométrie osseuse de référence de chaque patella, par segmentation sur chaque coupe axiale inframillimétrique à l'aide d'un logiciel spécifique (AVIZO v7.1.0, VSG SAS). Les géométries issues de l'algorithme de reconstruction biplanaire et de la segmentation scannographique étaient comparées par superposition des 2 modèles 3D de patella.

\subsubsection{Analyse de la précision et reproductibilité}

On réalisait le protocole biplanaire avec les pièces munies de billes. Deux opérateurs entraînés à la reconstruction biplanaire (LD et $\mathrm{BE}$ ) ont réalisé indépendamment 3 séquences de reconstruction et de recalage de la position relative des billes et de la patella afin de caractériser les incertitudes pour chaque degré de liberté (Fig. 6). La position exacte de la patella était estimée par la position moyenne des billes comme référence. Ainsi, la précision était évaluée par la comparaison des positions des billes et des positions relatives de la patella. La reproductibilité était évaluée par la variation des positions entre opérateurs.

\subsection{Analyse statistique}

L'analyse statistique était réalisée avec le logiciel MATLAB. Les différences et variations de positions relatives étaient exprimées pour deux écart-types, correspondant à l'IC 95\% et définissant 

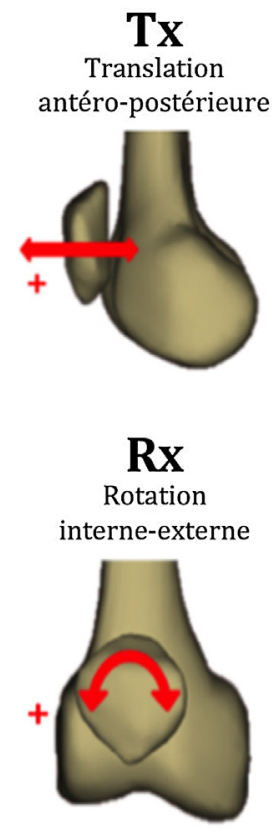
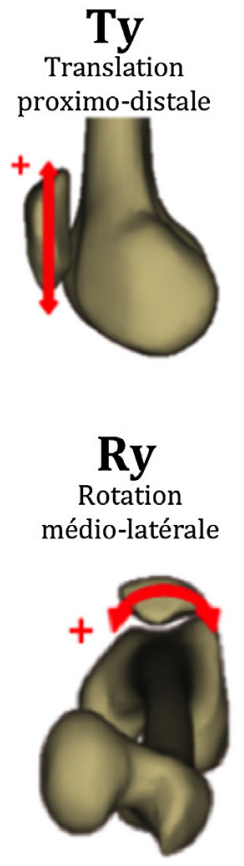

Tz

Translation médio-latérale

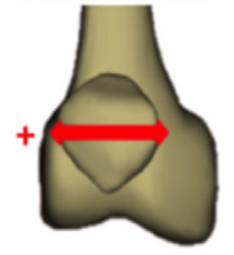

Rz

Rotation

flexion-extension

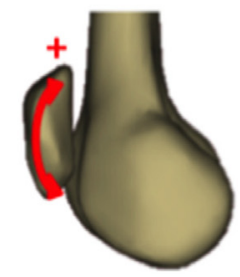

Fig. 4. Description des 6 degrés de liberté patellaires. La translation latérale est signée positive. La rotation latérale est signée négative.

l'incertitude. L'analyse de reproductibilité intégrait la variabilité du recalage de la patella pour chaque degré de liberté (norme ISO 5725:2). La comparaison de géométrie osseuse entre les objets 3D utilisait la méthode des moindres carrés et comprenait les erreurs moyennes, maximales et IC $95 \%$ de l'ensemble des distances pointssurfaces entre les 2 objets 3D.

\section{Résultats}

\subsection{Comparaison entre mouvement continu et séquentiel}

Les 6 cycles continus de flexion-extension présentaient des variations de translation et rotation inférieures à $0,5 \mathrm{~mm}$ et $0,5^{\circ}$ (Fig. 7). Les différences étaient inférieures à $1 \mathrm{~mm}$ et à $1^{\circ}$ entre la position séquentielle des tripodes et l'enregistrement continu des tripodes.

\subsection{Validation du gold standard par billes métalliques}

Le Tableau 1 rapporte la reproductibilité de l'enregistrement des 3 billes métalliques. L'IC $95 \%$ était de l'ordre de $0,1 \mathrm{~mm}$ et $0,5^{\circ}$ pour l'ensemble des degrés de liberté, validant notre méthode de mesure positionnelle comme méthode de référence.

\subsection{Précision et reproductibilité de la position de la patella par rapport au fémur}

L'étude de reproductibilité et de précision a porté sur la reconstruction de 4 membres inférieurs par 2 opérateurs trois fois chacun (soit 24 reconstructions biplanaires de fémur et patella) et le recalage patellaire des 4 positions de flexion supplémentaires (soit 36 phases de recalage). Les IC 95\% pour chaque degré de liberté patellaire sont reportés dans le Tableau 1 . Un exemple de comparaison entre les variations de recalage et la position de référence des billes est rapporté dans le Tableau 2.

\subsection{Analyse de la géométrie patellaire par méthode de reconstruction}

L'analyse des distances point/surface entre la géométrie issue des méthodes de reconstruction $3 \mathrm{D}$ et celle issue de la segmentation scannographique présentait une erreur moyenne de $0,49 \mathrm{~mm}$ avec un IC $95 \%=1,32 \mathrm{~mm}(\max : 2,78 \mathrm{~mm})$.

\section{Discussion}

Deux axes de validation ont été définis, avec la comparaison du mouvement continu et séquentiel, d'une part, et la quantification de la précision et de la reproductibilité de la position patellaire, d'autre part, en incluant la précision de la géométrie 3D de la patella. Cette validation cinématique s'appuie sur l'utilisation de deux gold standards : évaluation par tripodes infrarouges couplés à un système optoélectronique et par billes métalliques. Nous avons considéré le système optoélectronique comme système de mesure continue de référence par sa fiabilité $[6,7,14]$. Mais la superposition des tripodes infrarouges (peu représentative de conditions in vivo) rendait délicate l'acquisition des contours et la phase de recalage patellaire. L'utilisation de billes métalliques permettait de supprimer l'encombrement et la superposition dus aux tripodes et d'être utilisé comme référentiel de position après en avoir vérifié la fiabilité (répétabilité de l'ordre de $0,1 \mathrm{~mm}$ et $0,5^{\circ}$ dans cette étude).

Le simulateur du genou utilisé doit tout d'abord permettre la production de cycles de flexion-extension homogènes, pour que l'analyse cinématique continue soit pertinente et au plus proche de sa description in vivo. Les choix conceptuels de ce simulateur, explicités par Azmy et al. [14], permettent une simulation de cycles fiables et reproductibles : fémur fixe et tibia libre, ligaments tibio-fibulaires et membrane interosseuse conservés, sollicitation du tendon quadricipital unidirectionnelle et confondue avec l'axe fémoral mécanique. Ce choix permet de limiter les erreurs expérimentales dues à une approximation de la participation des muscles vaste médial, vaste latéral, vaste intermédiaire et droit fémoral, et la direction et la force de leurs actions modifient peu la cinématique patellaire dans des conditions expérimentales $[11,20]$. Le simulateur de flexion élaboré pour cette étude présentait une 


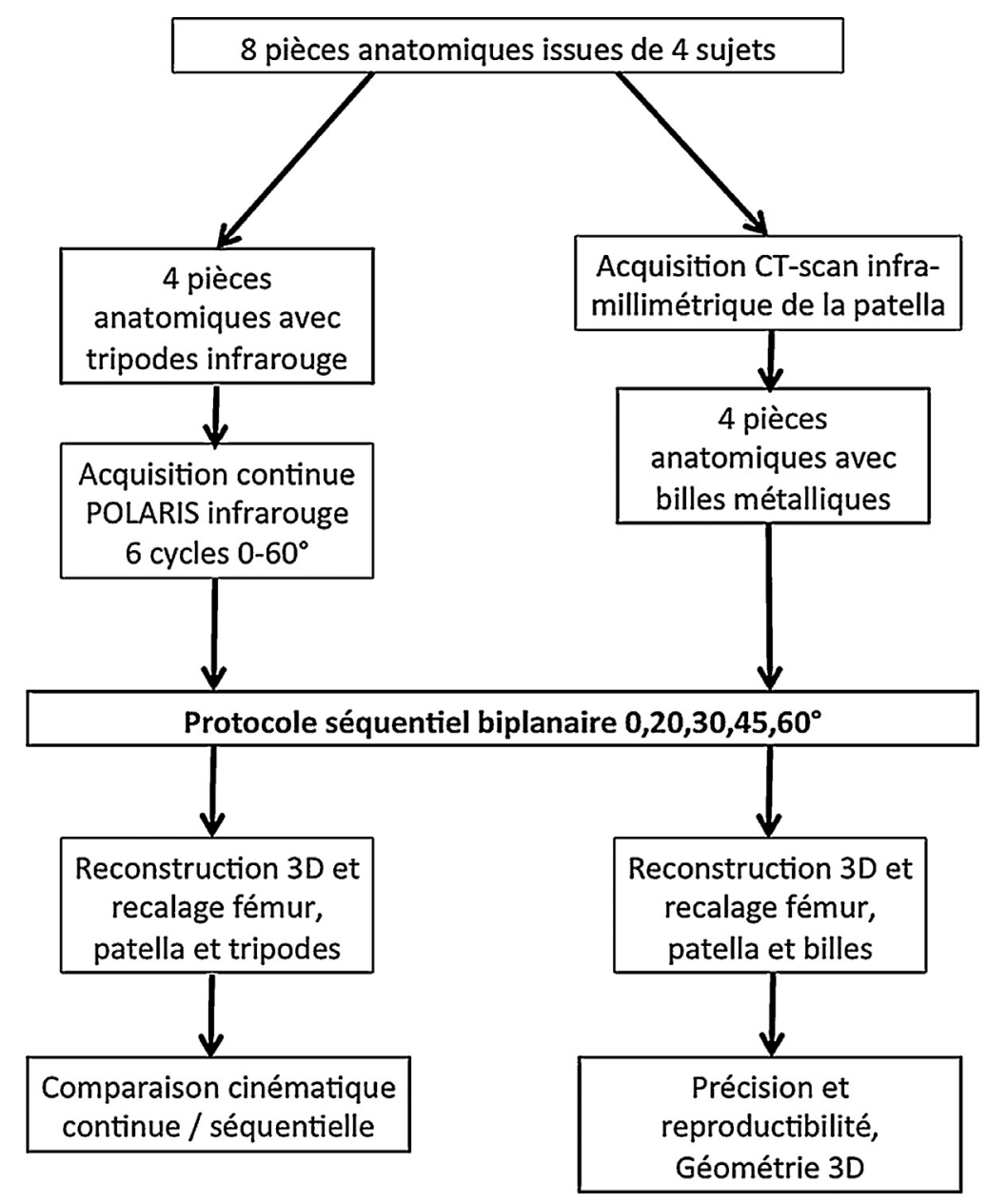

Fig. 5. Protocole de validation in vitro.

Tableau 1

Estimation de l'IC $95 \%$ concernant la reproductibilité et la précision en fonction du degré de liberté patellaire.

\begin{tabular}{|c|c|c|c|c|c|c|}
\hline \multirow[t]{2}{*}{ IC $95 \%$} & \multicolumn{3}{|c|}{ Translations (mm) } & \multicolumn{3}{|c|}{ Rotations $\left({ }^{\circ}\right)$} \\
\hline & $\mathrm{Tx}$ & Ty & $\mathrm{Tz}$ & $\mathrm{Rx}$ & Ry & $\mathrm{Rz}$ \\
\hline Reproductibilité de l'enregistrement des billes métalliques & 0,1 & 0,1 & 0,1 & 0,5 & 0,6 & 0,5 \\
\hline Précision de position de la patella par rapport au fémur & 0,8 & 0,7 & 1,7 & 6,5 & 2,5 & 1 \\
\hline Reproductibilité de la position de la patella par rapport au fémur & 1,1 & 1 & 2,2 & 5,8 & 5 & 2 \\
\hline
\end{tabular}

Tableau 2

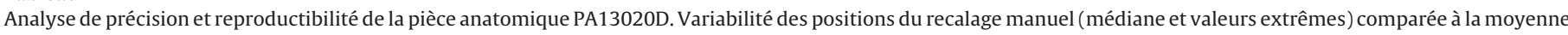
des positions des billes (référence) pour chaque degré de liberté et de flexion.

\begin{tabular}{|c|c|c|c|c|c|c|}
\hline & $\mathrm{Tx}(\mathrm{mm})$ & Ty $(\mathrm{mm})$ & $\mathrm{Tz}(\mathrm{mm})$ & $\operatorname{Rx}\left({ }^{\circ}\right)$ & $\operatorname{Ry}\left({ }^{\circ}\right)$ & $\mathrm{Rz}\left({ }^{\circ}\right)$ \\
\hline \multicolumn{7}{|l|}{$0^{\circ}$} \\
\hline Recalage & $50,6[50,51]$ & $18,4[18,20]$ & $8,5[7,10]$ & $8,2[5,12]$ & $-7,3[-6,-8]$ & $-0,5[0,-2]$ \\
\hline Billes & 50,6 & 18,4 & 8,5 & 8,2 & $-7,3$ & $-0,5$ \\
\hline \multicolumn{7}{|l|}{$20^{\circ}$} \\
\hline Recalage & $52,3[52,53]$ & $4,6[4,6]$ & $8,6[6,9]$ & $6,4[1,8]$ & $-7,5[-6,-13]$ & $-9,5[-8,-11]$ \\
\hline Billes & 52,5 & 4,4 & 7,7 & 7,6 & $-5,9$ & $-8,4$ \\
\hline \multicolumn{7}{|l|}{$30^{\circ}$} \\
\hline Recalage & $50,8[51,51]$ & $-2,1[-1,-3]$ & $8[6,9]$ & $6,4[4,11]$ & $-5,9[-4,-12]$ & $-14,4[-14,-16]$ \\
\hline Billes & 50,9 & $-2,2$ & 6,9 & 8,6 & $-4,4$ & $-13,7$ \\
\hline \multicolumn{7}{|l|}{$45^{\circ}$} \\
\hline Recalage & $45,7[45,46]$ & $-11,7[-11,-12]$ & $6,6[5,8]$ & $6,1[3,12]$ & $-4,9[-4,-9]$ & $-23,6[-22,-25]$ \\
\hline Billes & 45,9 & $-11,9$ & 4,9 & 8,4 & $-4,9$ & $-23,7$ \\
\hline \multicolumn{7}{|l|}{$60^{\circ}$} \\
\hline Recalage & $38,6[38,39]$ & $-19[-18,-20]$ & $6,5[5,7]$ & $4,1[2,9]$ & $-7,1[-4,-11]$ & $-37,7[-36,-40]$ \\
\hline Billes & 38,9 & $-18,9$ & 4,5 & 7,6 & -7 & $-37,6$ \\
\hline
\end{tabular}



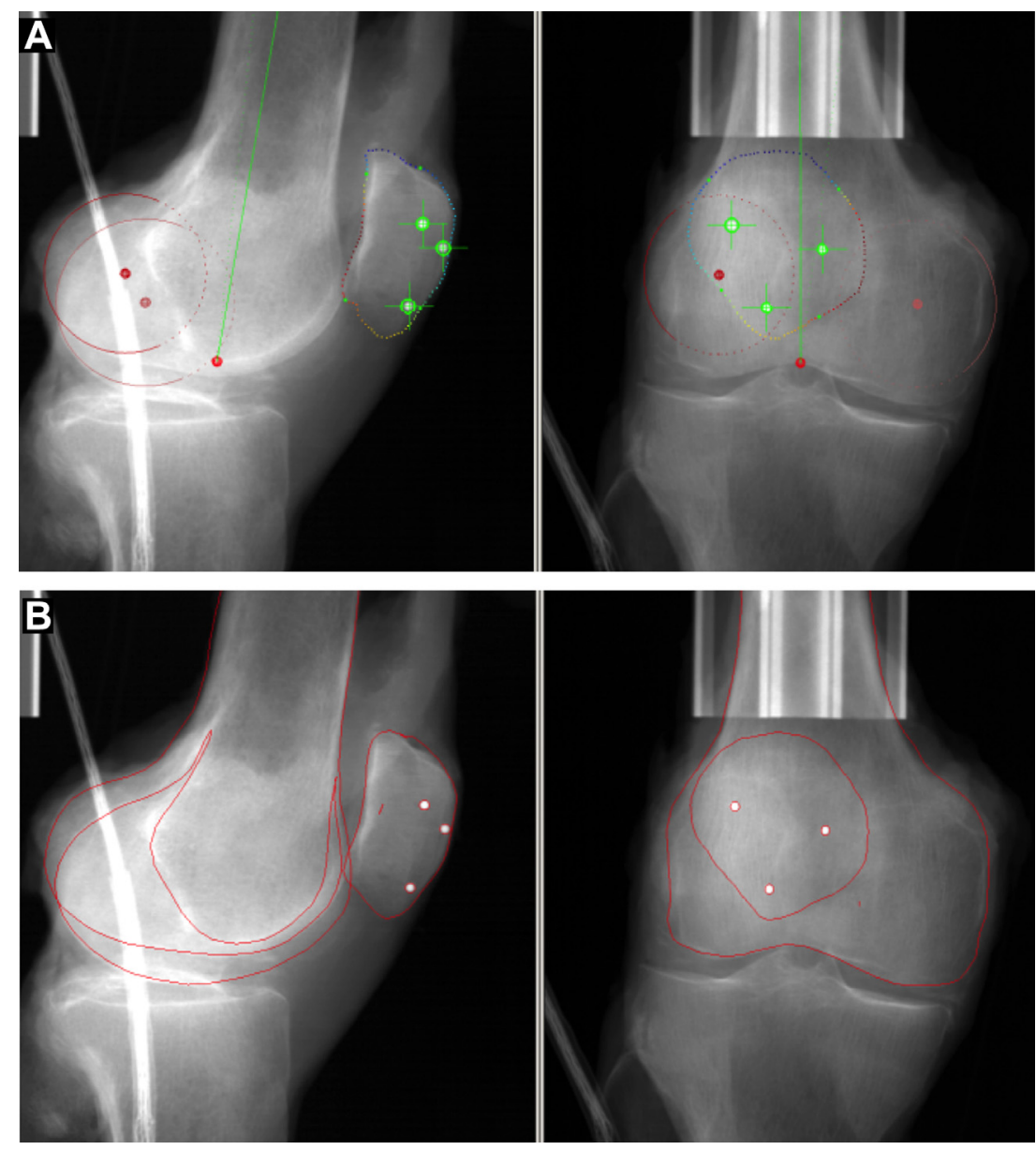

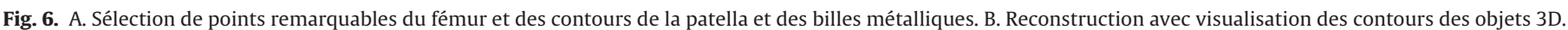

excellente reproductibilité des cycles de flexion-extension au sein d'une même pièce anatomique, primordial avant de pouvoir comparer cinématique continue et séquentielle. De plus, notre étude retrouve des différences positionnelles faibles (de l'ordre du degré et du millimètre) entre les mesures du mouvement continu et séquentiel. Cette différence est probablement minimisée in vitro comparativement aux conditions de charge in vivo et du tonus musculaire. La cinématique FP des différents cycles explorée par système optoélectronique est semblable à la description de la cinématique FP in vivo, avec des paramètres dépendants du degré de flexion du genou ( $\mathrm{Tx}$, Ty et $\mathrm{Rz}$ ) et des paramètres indépendants (Tz, Rx, et Ry) [7]. Dans cette étude, la course patellaire présentait une légère translation médiale (ou shift médial) ainsi qu'une rotation médiale (ou tilt médial) observable durant les premiers degrés de flexion, compatibles avec d'autres descriptions cinématiques $[7,10,21]$. Les profils cinématiques retrouvés dans notre étude sont donc cohérents avec d'autres protocoles de préparation et d'analyse in vitro. Nous avons choisi de décrire la cinématique séquentielle de la patella par rapport au fémur d'abord pour une raison technique (le banc d'essai prévoit une analyse avec un fémur fixe) et ensuite la majorité des études adopte cette description de manière conventionnelle [22,23]. Dans le cas d'une analyse in vivo, les déplacements du fémur et de la patella devront être pris en compte indépendamment avant d'en déduire la cinématique FP globale.

La précision de la position patellaire variait dans cette étude de 0,7 à $1,7 \mathrm{~mm}$ pour les translations. Les rotations dans le plan sagittal ( $\mathrm{Rz})$ et axiale (Ry) présentaient également une bonne précision (respectivement $2,5^{\circ}$ et $1^{\circ}$ ). Seule la rotation frontale $\mathrm{Rx}$ présentait une précision moindre (autour de $6^{\circ}$ ), par difficulté de saisie des contours patellaires de face et par l'absence de critères morphologiques identifiables. L'analyse du tracking in vivo en conditions pathologiques devra prendre en compte ces valeurs de précision [15]. L'analyse de la cinématique articulaire du genou par imagerie biplanaire a déjà fait l'objet de publications [15,24-26] mais l'originalité de notre méthode est d'utiliser uniquement la géométrie issue du contourage (modèles 3D personnalisés) sans imagerie préalable. Les acquisitions scannographiques de ce protocole ont été utilisées uniquement dans l'évaluation géométrique. Bey et al. [15] rapportent une excellente précision, inférieure à $0,5 \mathrm{~mm}$ et à $1^{\circ}$, mais leur méthode d'acquisition nécessite une analyse volumétrique haute-résolution du fémur et de la patella par scanner en y associant une phase de reconstruction. Sharma et al. [25] présentent des résultats similaires, dépendant de l'analyse scanner préalable et de l'utilisation d'une cage de calibration. Un protocole utilisable en routine clinique doit être le plus simple et accessible possible, sans imagerie préalable et en réduisant au maximum les phases de traitement d'images et de données.

La reproductibilité des protocoles est peu référencée dans la littérature [7,22], mais est fondamentale en cas de traitement des données par acquisition manuelle (saisie des contours, phase de recalage). Dans ce protocole, la variabilité intra-opérateur est pertinente dans le cadre des translations et de Rz. Elle est plus faible en Rx et Ry. Cette perte de reproductibilité est expliquée par la difficulté de saisie des contours patellaires et de la phase de recalage pour les 2 dernières positions ( 45 et $60^{\circ}$ de flexion), du fait de la projection avec le fémur et de la difficulté à saisir la pointe de la patella. Le recalage selon $\mathrm{Rx}$ sur la vue de face est d'autant plus difficile que la patella présente une flexion importante et cette variabilité influence la reproductibilité associée aux autres rotations. Sur la vue de profil, la difficulté est également caractérisée 

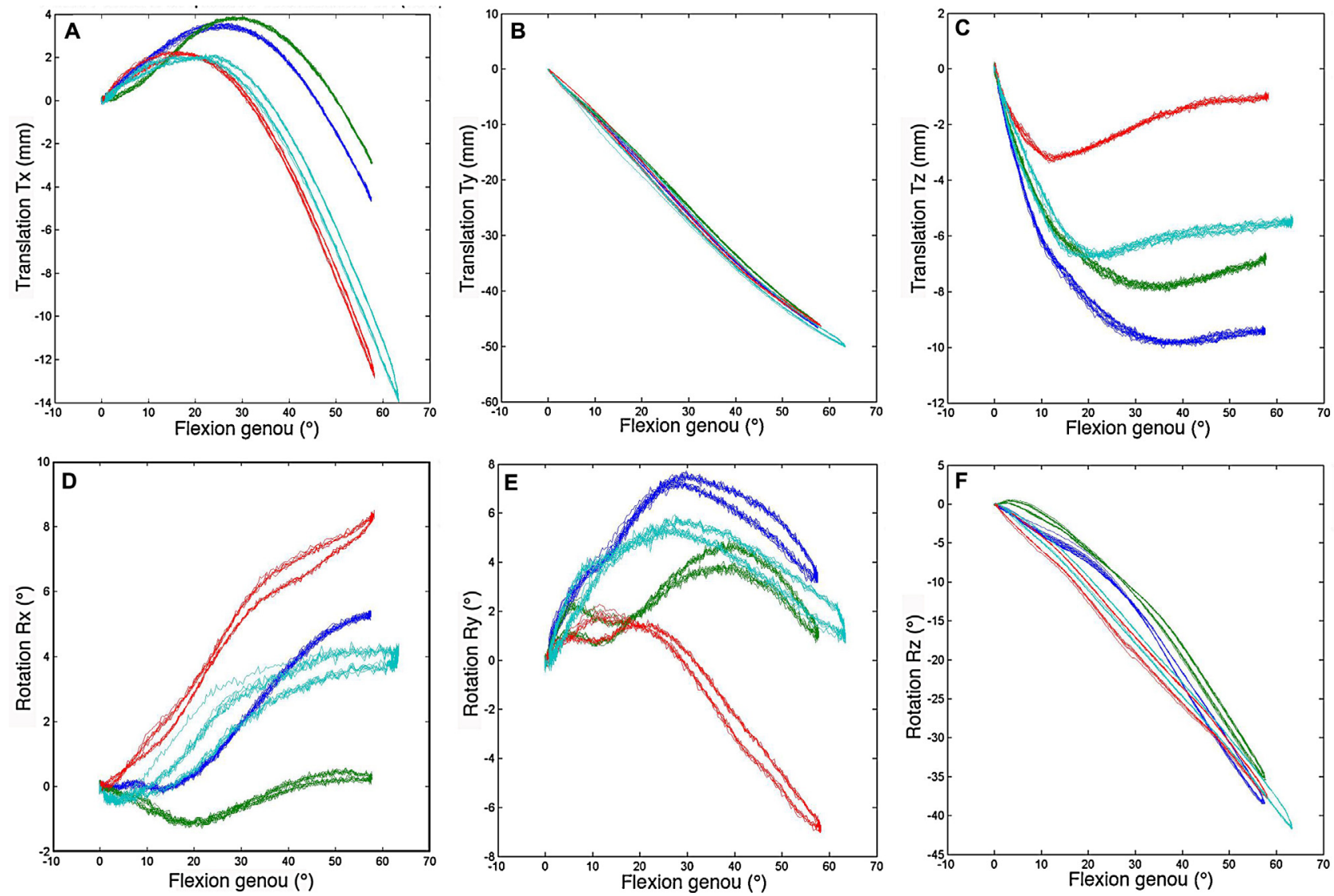

Fig. 7. A-F. Cinématique continue de la position 3D de la patella relative au fémur par système optoélectronique pour chaque degré de liberté.

par l'absence de critères identifiables. L'analyse du tracking in vivo devrait donc se limiter à son évaluation dans les premiers degrés de flexion du genou $\left(0-45^{\circ}\right)$ car l'incertitude peut augmenter en cas de positions extrêmes. En cas de pathologies FP, les variations cinématiques interviennent dans les trente premiers degrés de flexion, et peuvent présenter des différences relatives (de l'ordre de $5^{\circ}$ de bascules en moyenne pour les syndromes douloureux) $[4,27]$.

L'utilisation de contours biplanaires sans imagerie préalable est un challenge méthodologique du fait de la petite taille et de l'absence de critères anatomiques fiables de la patella. L'erreur moyenne de géométrie 3D était inférieure au millimètre sur les 4 patellae étudiées. Cette géométrie est primordiale car elle conditionne la description du référentiel patellaire par régionalisation de zones d'intérêt (la facette médiale, la facette latérale et la pointe). Elle influence également la précision et la reproductibilité du protocole [9]: moins la géométrie est fiable, plus on augmente les incertitudes autour de sa position séquentielle. L'effet de la géométrie patellaire sur le système de coordonnées devra être apprécié en cas d'altération morphologique importante (dysplasie essentiellement). Aucune pièce anatomique évaluée ne présentait de patella dysplasique dans ce travail. De plus, les erreurs maximales de géométrie se situaient au niveau de la berge latérale, alors que l'hypoplasie siège sur le versant médial de la patella. Cette incertitude relative aux dysplasies devra donc faire l'objet d'une étude plus spécifique.

Ce protocole de validation in vitro présente certaines limites. La première est le faible nombre de pièces anatomiques étudiées dans chaque axe de validation, provenant de prélèvements bilatéraux. La variabilité des profils séquentiels peut s'expliquer par une variabilité inter-spécimens, due à des différences morphologiques ou cinématiques. Une concordance anatomique est généralement retrouvée entre prélèvements bilatéraux [28], et les variations morphologiques du fémur distal intéressent principalement la partie antérieure des condyles fémoraux et les contours de la trochlée [14,22]. Notre choix s'est donc porté sur la morphologie des condyles postérieurs comme référence pour la construction du repère anatomique "fémur ". Concernant les variabilités d'origine patellaires, la cinématique et la géométrie de la patella ne paraissent pas symétriques en cas d'évaluation bilatérale [29]. Les variations maximales entre cinématique droite et gauche étaient respectivement de $2,14^{\circ} ; 0,46^{\circ}$; et $1,30 \mathrm{~mm}$ pour les rotations, bascules et translations, même si aucune différence significative n'a pu être notée [2]. Une deuxième limite est représentée par le modèle in vitro. Il permet uniquement de reproduire un mouvement passif de flexion du genou, et reste une approximation du comportement dynamique in vivo des facteurs de stabilisation actifs de la patella $[22,30]$.

Mais nous proposons une nouvelle approche séquentielle de la course FP in vitro, à l'aide de méthodes de reconstruction 3D innovantes et d'un système d'imagerie ne cessant d'élargir son champ d'activité. De plus, l'absence d'imagerie préalable (de type scanner ou IRM) ainsi que le caractère peu irradiant de ce protocole sont des arguments en faveur d'une évaluation réalisable in vivo. Par la description rigoureuse des phases de reconstruction et de recalage, cette analyse doit être accessible à tout clinicien ou technicien formé à la reconstruction biplanaire, comme c'est le cas actuellement pour d'autres analyses fonctionnelles et mesures EOS. Enfin, les perspectives cliniques FP sont nombreuses : définir le profil cinématique FP d'un genou asymptomatique, évaluer les différences positionnelles en cas d'instabilité FP, voire quantifier l'effet postopératoire d'une chirurgie de correction sur la cinématique patellaire en charge. 


\section{Déclaration de lien d'intérêts}

L.D: subvention de la SO.F.C.O.T durant la conduite de l'étude. P.T, B.E et F.C. déclarent ne pas avoir de liens d'intérêts.

W.S. : subvention de la fondation ParisTech durant la conduite de l'étude. Co-inventeur du système EOS, sans aucun bénéfice financier individuel.

\section{Remerciements}

Ce travail a été soutenu par la SO.F.C.O.T par l'intermédiaire de la bourse "Année Recherche 2013 ».

Les auteurs remercient également les partenaires du programme de Chaire ParisTech BiomecAM en modélisation musculosquelettique personnalisée, et en particulier la fondation ParisTech, la Société Générale et le groupe COVEA, ainsi que Benjamin Aubert et Thomas Joubert de l'institut de biomecanique humaine GeorgesCharpak pour leur support technique.

\section{Références}

[1] Draper CE, Besier TF, Fredericson M, Santos JM, Beaupre GS, Delp SL, et al. Differences in patellofemoral kinematics between weight-bearing and nonweight-bearing conditions in patients with patellofemoral pain. J Orthop Res 2011:29:312-7.

[2] MacIntyre NJ, Hill NA, Fellows RA, Ellis RE, Wilson DR. Patellofemoral joint kinematics in individuals with and without patellofemoral pain syndrome. J Bone Joint Surg Am 2006;88:2596-605, http://dx.doi.org/10.2106/JBJS.E.00674.

[3] Harman M, Dogan A, Arslan H, Ipeksoy U, Vural S. Evaluation of the patellofemoral joint with kinematic MR fluoroscopy. Clin Imaging 2002;26: 136-9.

[4] Song C-Y, Lin J-J, Jan M-H, Lin Y-F. The role of patellar alignment and tracking in vivo: the potential mechanism of patellofemoral pain syndrome. Phys Ther Sport 2011;12:140-7.

[5] Cheung RTH, Mok NW, Chung PYM, Ng GYF. Non-invasive measurement of the patellofemoral movements during knee extension-flexion: a validation study. Knee 2013;20:213-7.

[6] Jenny JY, Lefebvre Y, Vernizeau M, Lavaste F, Skalli W. In vitro optoelectronic analysis of the continuous active knee kinematics. Rev Chir Orthop Reparatrice Appar Mot 2002;88:790-6.

[7] Philippot R, Chouteau J, Testa R, Moyen B. In vitro analysis of patellar kinematics: validation of an opto-electronic cinematic analysis protocol. Knee Surg Sports Traumatol Arthrosc 2009;18:161-6.

[8] Belvedere C, Leardini A, Ensini A, Bianchi L, Catani F, Giannini S Three-dimensional patellar motion at the natural knee during passive flexion/extension. An in vitro study. J Orthop Res 2009;27:1426-31.

[9] Ostermeier S, Holst M, Bohnsack M, Hurschler C, Stukenborg-Colsman C, Wirth C-J. In vitro measurement of patellar kinematics following reconstruction of the medial patellofemoral ligament. Knee Surg Sports Traumatol Arthrosc 2006;15:276-85.

[10] Amis AA, Senavongse W, Bull AMJ. Patellofemoral kinematics during knee flexion-extension: an in vitro study. J Orthop Res 2006;24:2201-11.
[11] Lorenz A, Müller O, Kohler P, Wünschel M, Wülker N, Leichtle UG. The influence of asymmetric quadriceps loading on patellar tracking - an in vitro study. Knee 2012;19:818-22.

[12] Ahmed AM, Duncan NA, Tanzer M. In vitro measurement of the tracking pattern of the human patella. J Biomech Eng 1999;121:222-8.

[13] Nagamine R, Otani T, White SE, McCarthy DS, Whiteside LA. Patellar tracking measurement in the normal knee. J Orthop Res 1995;13:115-22.

[14] Azmy C, Guérard S, Bonnet X, Gabrielli F, Skalli W. EOS orthopaedic imaging system to study patellofemoral kinematics: assessment of uncertainty. Orthop Traumatol Surg Res 2010;96:28-36.

[15] Bey MJ, Kline SK, Tashman S, Zauel R. Accuracy of biplane x-ray imaging combined with model-based tracking for measuring in-vivo patellofemoral joint motion. J Orthop Surg 2008;3:38.

[16] Laporte S, Skalli W, De Guise JA, Lavaste F, Mitton D. A biplanar reconstruction method based on 2D and 3D contours: application to the distal femur. Comput Methods Biomech Biomed Engin 2003;6:1-6.

[17] Chaibi Y, Cresson T, Aubert B, Hausselle J, Neyret P, Hauger O, et al. Fast 3D reconstruction of the lower limb using a parametric model and statistical inferences and clinical measurements calculation from biplanar X-rays. Comput Methods Biomech Biomed Engin 2012;15:457-66.

[18] Quijano S, Serrurier A, Aubert B, Laporte S, Thoreux P, Skalli W. Threedimensional reconstruction of the lower limb from biplanar calibrated radiographs. Med Eng Phys 2013;35:1703-12.

[19] Grood ES, Suntay WJ. A joint coordinate system for the clinical description of three-dimensional motions: application to the knee. J Biomech Eng 1983;105:136-44

[20] Elias JJ, Bratton DR, Weinstein DM, Cosgarea AJ. Comparing two estimations of the quadriceps force distribution for use during patellofemoral simulation. J Biomech 2006;39:865-72.

[21] Zaffagnini S, Colle F, Lopomo N, Sharma B, Bignozzi S, Dejour D, et al. The influence of medial patellofemoral ligament on patellofemoral joint kinematics and patellar stability. Knee Surg Sports Traumatol Arthrosc 2012:21:2164-71.

[22] Katchburian MV, Bull AMJ, Shih Y-F, Heatley FW, Amis AA. Measurement of patellar tracking: assessment and analysis of the literature. Clin Orthop 2003;412:241-59.

[23] Bull A, Katchburian M, Shih Y-F, Amis A. Standardisation of the description of patellofemoral motion and comparison between different techniques. Knee Surg Sports Traumatol Arthrosc 2002;10:184-93.

[24] Baka N, Kaptein BL, Giphart JE, Staring M, Bruijne M, de Lelieveldt BPF, et al. Evaluation of automated statistical shape model based knee kinematics from biplane fluoroscopy. J Biomech 2014;47:122-9.

[25] Sharma GB, Saevarsson SK, Amiri S, Montgomery S, Ramm H, Lichti DD, et al. Radiological method for measuring patellofemoral tracking and tibiofemoral kinematics before and after total knee replacement. Bone Joint Res 2012;1:263-71.

[26] Giphart JE, Zirker CA, Myers CA, Pennington WW, LaPrade RF. Accuracy of a contour-based biplane fluoroscopy technique for tracking knee joint kinematics of different speeds. J Biomech 2012;45:2935-8.

[27] Powers CM. Patellar kinematics, part II: the influence of the depth of the trochlear groove in subjects with and without patellofemoral pain. Phys Ther 2000;80:965-73.

[28] Dargel J, Feiser J, Gotter M, Pennig D, Koebke J. Side differences in the anatomy of human knee joints. Knee Surg Sports Traumatol Arthrosc 2009;17:1368-76, http://dx.doi.org/10.1007/s00167-009-0870-5.

[29] Laprade J, Lee R. Real-time measurement of patellofemoral kinematics in asymptomatic subjects. Knee 2005;12:63-72.

[30] Brossmann J, Muhle C, Schröder C, Melchert UH, Büll CC, Spielmann RP, et al. Patellar tracking patterns during active and passive knee extension: evaluation with motion-triggered cine MR imaging. Radiology 1993;187:205-12. 